\title{
Efecto de tres marcadores genéticos sobre la eficiencia alimenticia de toretes en prueba de comportamiento
}

\section{Effect of three genetic markers on feeding efficiency of yearling bulls under performance test}

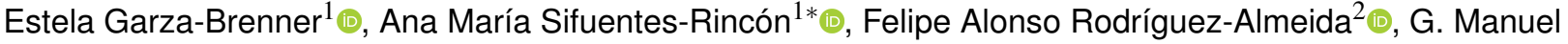 \\ Parra-Bracamonte ${ }^{1}$ (D), Williams Arellano-Vera ${ }^{1}$ (D) \\ ${ }^{1}$ Laboratorio de Biotecnología Animal, Centro de Biotecnología Genómica, Instituto Politécnico Nacional. Blvd. Del maestro esq. Elias \\ Piña, Col. Narciso Mendoza s/n, CP. 88710. Ciudad Reynosa, Tamaulipas, México. \\ ${ }^{2}$ Facultad de Zootecnia y Ecología. Universidad Autónoma de Chihuahua. Periférico Francisco R. Almada km 1. CP. 31453. Chihuahua, \\ Chihuahua, México. \\ *Autor de correspondencia: asifuentes@ipn.mx
}

Nota científica recibida: 12 de febrero de 2019 aceptada: 17 de abril de 2019

RESUMEN. Se evaluó el efecto de tres marcadores genéticos localizados en los genes de hormona de crecimiento $(\mathrm{GH})$, factor de crecimiento semejante a insulina-1 (IGF-1) y leptina (LEP) sobre indicadores de eficiencia alimenticia, utilizando registros de 136 toretes de las razas Charolais, Angus y Brangus durante una prueba de comportamiento productivo. EI análisis de asociación entre los genotipos obtenidos y los rasgos productivos evaluados mostró un efecto significativo del marcador GH-Alu sobre CRA ( $p=0.032)$ en la raza Angus, y sobre el peso final ajustado $(p=0.041)$, en la Charolais; este resultado muestra que es posible su inclusión en los esfuerzos iniciales para la determinación de fenotipos de eficiencia en México y su consecuente aplicación en las estrategias de selección y mejoramiento genético.

Palabras clave: Bovinos de carne, consumo residual, GH, IGF-1, LEP, polimorfismo de un solo nucleótido.

ABSTRACT. The effect of three molecular markers located at growth hormone (GH), insulin-like growth factor-1 (IGF-1) and leptin (LEP) genes on feed efficiency traits was evaluated in 136 young bulls Charolais, Angus and Brangus cattle breeds under a performance test The association analysis showed significant effect of GH-Alu marker on RFI of Angus animals ( $p$ $=0.032)$ and adjusted final weight of Charolais animals $(p=0.041)$. This result is important because demonstrates that it is possible its application on the initial efforts focused on the analysis of feeding efficiency phenotypes in Mexico, allowing their future use in selection and genetic improvement.

Key words: Beef cattle, GH, IGF-1, LEP, single nucleotide polymorphism.

\section{INTRODUCCIÓN}

El componente más costoso en los sistemas de producción animal corresponde a los gastos de alimentación, en los que se invierte entre el 60 y $75 \%$ de los costos totales de producción (Crews et al. 2005, Alende 2017). Por tal razón, la inclusión de criterios que permitan precisar la identificación y selección de animales con mayor potencial para lograr la óptima utilización del alimento con rendimientos económicos superiores, es un tema relevante no solo desde el punto de vista económico y productivo, sino también podría tener un impacto ambiental, ya que se ha re- portado que el ganado que es más eficiente emite cantidades más bajas de metano (Llonch et al. 2016).

Generalmente, al llevar a cabo estudios sobre el análisis de eficiencia alimenticia en especies de importancia económica se busca relacionar el consumo de alimento con la productividad del animal o del hato. El consumo residual de alimento (CRA), se define como la diferencia entre el consumo de alimento real y el consumo esperado, por lo que los animales identificados como eficientes, serán aquellos que consuman una menor cantidad de alimento al esperado para su peso corporal y tasa de crecimiento; de igual manera, los animales considerados como ineficientes 
serán los que consuman mayor cantidad de alimento al esperado (Koch et al. 1963). Uno de los principales problemas para la adopción de las diferentes pruebas para determinar la eficiencia alimenticia de un hato bovino es la dificultad técnica y los elevados costos para medir el rasgo, lo que hace que este fenotipo sea candidato óptimo para la implementación de las estrategias de selección asistida por marcadores para la identificación de individuos superiores en conversión alimenticia (Crews et al. 2005, Sherman et al. 2008).

La regulación fisiológica de la ingesta de alimento, crecimiento y la distribución de energía en animales está bajo el control de múltiples genes, que se han considerado como candidatos para la selección asistida. Los polimorfismos localizados en los genes de la hormona de crecimiento $(\mathrm{GH})$, factor de crecimiento semejante a insulina tipo 1 (IGF-1) y leptina (LEP), han sido ampliamente estudiados y asociados a diferentes rasgos productivos entre los que se encuentra la eficiencia alimenticia (Sherman et al. 2008). La hormona de crecimiento (GH) ha recibido especial interés ya que influye de manera directa (efecto metabólico) o indirecta (efecto somatogénico) en una gran variedad de tejidos y procesos fisiológicos, como el crecimiento, contenido de grasa de la canal y músculo, consumo de alimento y conversión alimenticia (Pereira et al. 2005, Banos et al. 2008). De los polimorfismos localizados en el gen de $\mathrm{GH}$, el ubicado en la posición 127 del exón 5 es de los más estudiados. Este Polimorfismo de un Solo Nucleótido (SNP) produce una sustitución de leucina (L) a valina (V) y se puede caracterizar usando la enzima de restricción Alu I, por lo que se identifica como marcador $\mathrm{GH}-A$ lu I; Junto con el gen $\mathrm{GH}$, el gen IGF1, son reguladores importantes del conocido eje GH-IGF-1. La hormona IGF-1 regula el crecimiento y metabolismo celular y ha demostrado asociación con una mayor eficiencia alimenticia (Bishop et al. 1989, Stick et al. 1998). De los polimorfismos identificados en el gen IGF-1, es la transición de timina a citocina (T/C), también llamada RFLP-SnaBI (Ge et al. 2001), se ha asociado significativamente con rasgos productivos en varias razas de ganado (Wood et al. 2004, De la Rosa et al. 2010). Otro gen muy estudiado es el que codifica la leptina (LEP), que ha ganado gran atención como regulador clave de procesos biológicos que están relacionados con características productivas importantes en bovinos como la eficiencia alimenticia, el consumo de alimento, el contenido de grasa y la calidad de la carne (Geary et al. 2003, Nkrumah et al. 2005). Marcadores en el gen LEP ya forman parte de algunos paneles comerciales de marcadores genéticos diseñados para la selección asistida (MAS) en bovinos (Corva et al. 2009), uno de ellos es la transición de citosina (C) a timina (T) identificada en el exón 2 del gen que da como resultado un cambio aminoacídico de arginina a cisteína (Buchanan et al. 2002).

A pesar de su importancia, las pruebas de eficiencia alimenticia están en sus comienzos en México, por lo tanto, es importante que en los esfuerzos iniciales para fenotipificar estos rasgos ya se incluyan las nuevas herramientas destinadas a ayudar a la identificación de animales superiores. Por lo anterior el objetivo del estudio fue conocer el efecto de tres marcadores del tipo SNP's sobre rasgos de eficiencia alimenticia en toretes jóvenes de tres razas cárnicas, sometidos a las primeras pruebas de comportamiento realizadas en el Estado de Chihuahua.

\section{MATERIALES Y MÉTODOS}

El presente estudio no requirió la aprobación ética de algún Comité de Cuidado y Uso de Animales porque los datos se extrajeron de los libros de registro productivo y las muestras biológicas para el aislamiento del $A D N$ se obtuvieron del biobanco del Laboratorio de Biotecnología Animal del CBG-IPN.

Se incluyeron en el estudio toretes sometidos a dos pruebas de comportamiento para la evaluación de la eficiencia alimenticia, con base en el consumo residual de alimento. Las pruebas se realizaron en el Complejo Palomas de la Unión Ganadera Regional de Chihuahua (UGRCH). Un total de 136 animales de las razas Angus ( $A N=63)$, Brangus $(B R=41)$ y Charolais $(\mathrm{CH}=32)$, fueron incluidos en el estudio. La edad inicial promedio fue de $273 \pm 38 \mathrm{~d}$ con un peso corporal de $272 \pm 38 \mathrm{~kg}$. Los animales fueron sometidos a la misma dieta, la que contenía por ración 
$90.25 \%$ de materia seca, $13.25 \%$ de proteína cruda y 3.31 (Mcal $/ \mathrm{kg} \mathrm{MS}$ ) de energía diaria. Al arribo a las instalaciones, los animales tuvieron un período de adaptación de 20 días, y posteriormente 70 días de prueba. Los animales se pesaron dos días seguidos al inicio y al final del periodo de prueba, con pesajes cada 14 días en el transcurso de esta. La prueba se llevó a cabo utilizando el sistema automatizado de consumo GrowSafe ${ }^{\circledR}$ (GrowSafe System Ltd). A los animales se les colocó un arete electrónico en la oreja, con el cual se registró su presencia en el comedero y se midió la cantidad de alimento consumido en cada evento de consumo. Posteriormente se calculó el consumo de materia seca (CMS), consumo residual de alimento (CRA) de acuerdo con la metodología propuesta por Koch et al. (1963), conversión alimenticia (CA) y la ganancia diaria de peso (GDP), así como peso corporal: peso al destete ajustado a los 205 días de edad (PD) y peso final ajustado con regresión con todos los pesajes (PFAj).

Se colectaron muestras de pelo de los animales al inicio de las pruebas de comportamiento. Para la extracción del ADN se utilizó el kit comercial Genelute Mammalian Genomic DNA ${ }^{\circledR}$ (Cat. G1N350, Sigma-Aldrich Co). Se analizaron tres marcadores, localizados en cada gen candidato evaluado (GH, IGF-1 y LEP), utilizando ensayos de discriminación alélica. Cada ensayo, se realizó de forma individual en placas ópticas de 96 pozos en un equipo de PCR en tiempo real (ABI Prism 7500 Sequence Detection System $\left.{ }^{\circledR}\right)$, bajo las siguientes condiciones: un ciclo de $2 \min$ a $50^{\circ} \mathrm{C}$ y $10 \mathrm{~min}$ a 95 ${ }^{\circ} \mathrm{C}$, seguido de 40 ciclos de dos pasos $15 \mathrm{~s}$ a $92{ }^{\circ} \mathrm{C}$ y 1 min a $60^{\circ} \mathrm{C}$. La asignación de los genotipos se realizó mediante el paquete computacional (ABI Prism 7500 Real-Time Sequence Detection Software). Las frecuencias genotípicas y alélicas fueron analizadas en el programa GENEPOP versión en línea 4.0 (Rousset 2008).

Mediante un modelo lineal general se estimó el efecto de las razas evaluadas utilizando el siguiente modelo: $\mathrm{Y}_{i j k}=\mu+\mathrm{R}_{i}+\mathrm{H}_{j}+\varepsilon_{i j k}$, donde: $\mathrm{Y}_{i j k}=$ observación en el k-ésimo animal para cada una de las variables (CRA, CDMS, CA, GDP, PD y PFAj), $\mu=$ media general, $\mathrm{R}_{i}=$ efecto fijo de la raza en la prueba, $\mathrm{H}_{j}=$ efecto del periodo de la prueba de comportamiento y $\varepsilon_{i j k}=$ error aleatorio asociado al muestreo. Para probar el efecto de genotipo en los marcadores analizados para cada característica se utilizó el mismo modelo reemplazando el efecto de raza por el del marcador (GH-Alu, IGF-Sna BI y LEP). Las medias de los cuadrados mínimos fueron comparadas mediante una prueba de Tukey. Complementariamente, se realizó un análisis de correlación de Pearson. Todos los análisis fueron realizados en el paquete SAS versión 9.4.

\section{RESULTADOS Y DISCUSIÓN}

En la Tabla 1, se muestran los resultados de las medias por raza, para las variables de PD, PFAj, CMS, GDP, CA = CDMS/GDP y CRA, donde la raza que presentó los mayores pesos al destete fue la Brangus, mientras que para los pesos finales de la prueba la raza Charolais mostró los menores pesos; pero sin diferencias significativas entre las variables GDP y CRA en las tres razas. Se observó que la raza Angus presentó la mayor conversión alimenticia (CA $=6.69 \pm 0.10 ; p<0.05)$, demostrando que bajo las condiciones llevadas del experimento, las razas Brangus y Charolais son más eficientes en términos de conversión alimenticia, al requerir un menor consumo de alimento (6.15 y 5.91 vs 6.69) para desarrollar un kilogramo de peso vivo, aunque el Charolais alcanzó un menor PFAj a pesar de considerarse de mayor talla madura (CONARGEN 2017): para las variables de ganancias diarias de pesos promedios y consumo residual de alimento los resultados para las tres razas fueron similares.

La evaluación de CRA se ha convertido en una de las pruebas de elección para evaluar la eficiencia en el ganado de carne. Sin embargo, su desventaja como un indicador productivo más en las pruebas de comportamiento son los costos y la necesidad de equipo sofisticado para llevarla a cabo, lo que ha limitado su aplicación. Estas desventajas hacen a esta característica productiva un candidato ideal para el uso de la estrategia de selección asistida por marcadores moleculares.

Los tres marcadores genéticos relacionados 
Tabla 1. Media de las variables evaluadas de la prueba de eficiencia alimenticia entre razas.

\begin{tabular}{|c|c|c|c|c|c|c|}
\hline Raza & PD & PFAj & CDMS (kg) & GDP (kg) & CA & CRA (kg) \\
\hline Angus & $237.06 \pm 5.59^{b}$ & $417.50 \pm 5.55^{a}$ & $10.24 \pm 0.14^{a}$ & $1.55 \pm 0.02$ & $6.69 \pm 0.10^{a}$ & $0.002 \pm 0.09$ \\
\hline Brangus & $268.21 \pm 5.99^{a}$ & $432.26 \pm 6.88^{a}$ & $9.94 \pm 0.17^{a}$ & $1.63 \pm 0.03$ & $6.15 \pm 0.13^{b}$ & $-0.011 \pm 0.11$ \\
\hline Charolais & $217.20 \pm 7.12^{c}$ & $394.50 \pm 7.79^{b}$ & $9.00 \pm 0.20^{b}$ & $1.53 \pm 0.04$ & $5.91 \pm 0.15^{b}$ & $-0.165 \pm 0.13$ \\
\hline
\end{tabular}

con eficiencia alimenticia y características de importancia económica, se han utilizado para otras razas de carne, por lo que se conoce cuál de los dos alelos del polimorfismo se asocia con el mayor efecto en el rasgo productivo evaluado o alelo favorable (Pereira et al. 2005, Almeida et al. 2008, De la Rosa et al. 2010, Yurnalis y Putra 2017). En los animales analizados, las frecuencias alélicas de los marcadores GH-Alu I e IGF-1-Sna BI fueron mayores para el alelo $\mathrm{C}$, el cual en ambos marcadores ha sido reportado como el alelo favorable para características productivas. En el caso del marcador GH-Alu I, el alelo $C$ favorable se asocia con mayor marmoleo y peso de canal; mientras que el marcador IGF-1Sna BI se asocia con mayores pesos al destete (De la Rosa et al. 2010). Para el marcador LEP la frecuencia del alelo reportado como favorable fue más baja en la raza Charolais (Tabla 2). Este resultado, es importante ya que se sugiere que la sola frecuencia de los alelos reportados como favorables, en homocigosis e incluso heterocigosis representa un evento que podría favorecer la selección en el mejoramiento del ganado, conocer las frecuencias de estos alelos en poblaciones locales representa una ventaja para el establecimiento de estrategias para mantenerlas y evaluar su efecto en las características deseadas.

Los resultados del análisis de asociación de los tres marcadores considerados con las variables evaluadas durante la prueba de comportamiento para cada raza, determinó para Angus, una asociación significativa entre el marcador $\mathrm{GH}-\mathrm{Alu}(\mathrm{p}=0.032)$ para la variable $C R A$, donde los animales homocigotos $G G$ y CC (0.48 y 0.13 , respectivamente) resultaron ser menos eficientes que aquellos con los genotipos GC $(-0.26)$. El mismo marcador tuvo efecto significativo para PFAj $(p=0.040)$ en ganado Charolais (Tabla 3$)$.

Para esta variable, los portadores del genotipo
Tabla 2. Frecuencias genotípicas y alélicas de los marcadores en toretes de razas de bovinos de carne.

\begin{tabular}{cccc}
\hline Marcador & ANGUS & BRANGUS & CHAROLAIS \\
\hline LEP & $\mathrm{n}=56$ & $\mathrm{n}=33$ & $\mathrm{n}=25$ \\
${ }^{a} \mathrm{CC}$ & 0.18 & 0.06 & 0.36 \\
${ }^{a} \mathrm{CT}$ & 0.46 & 0.45 & 0.40 \\
${ }^{a} \mathrm{TT}$ & 0.36 & 0.49 & 0.24 \\
${ }^{b} \mathrm{C}$ & 0.41 & 0.28 & 0.56 \\
${ }^{b} \mathrm{~T}$ & 0.59 & 0.72 & 0.44 \\
$\mathrm{GH}-\mathrm{Alu}$ & $\mathrm{n}=43$ & $\mathrm{n}=38$ & $\mathrm{n}=28$ \\
${ }^{a} \mathrm{GG}$ & 0.12 & 0.08 & 0.18 \\
${ }^{a} \mathrm{GC}$ & 0.44 & 0.26 & 0.32 \\
${ }^{a} \mathrm{CC}$ & 0.44 & 0.66 & 0.50 \\
${ }^{b} \mathrm{G}$ & 0.34 & 0.21 & 0.34 \\
${ }^{b} \mathrm{C}$ & 0.66 & 0.78 & 0.66 \\
IGF-SnaB1 & $\mathrm{n}=61$ & $\mathrm{n}=41$ & $\mathrm{n}=29$ \\
${ }^{a} \mathrm{TT}$ & 0.03 & 0.17 & 0.07 \\
${ }^{a} \mathrm{TC}$ & 0.73 & 0.56 & 0.53 \\
${ }^{a} \mathrm{CC}$ & 0.25 & 0.27 & 0.40 \\
${ }^{b} \mathrm{~T}$ & 0.39 & 0.45 & 0.33 \\
${ }^{b} \mathrm{C}$ & 0.61 & 0.55 & 0.67 \\
\hline${ }^{a}=$ frecuencias genotípicas; ${ }^{b}=$ frecuencias alélicas.
\end{tabular}

CC (414 \pm 11.63$)$ fueron más pesados en contraste con la media de los genotipos GG y GC (382 \pm 22.06 y $362 \pm 16.44$, respectivamente). Este resultado es interesante, ya que una vez validado con mayor número de muestras, sería el primer reporte de asociación del marcador GH-Alu con CRA, ya que hasta la fecha de los genes que conforman el eje somatotrófico, solo se había reportado el efecto de un marcador localizado en un intrón del receptor de GH sobre este indicador de eficiencia (Sherman et al. 2008). Aunque no se ha encontrado un marcador de efecto mayor para el CRA, se espera que la combinación del efecto de los diferentes marcadores que se asocian a esta característica explique una gran proporción de la variación genética asociada a este rasgo, por lo que es importante que se realicen estudios para evaluar el efecto de estos marcadores en las poblaciones de ganado de carne. La correcta utilización de los registros productivos, su llenado periódico y actualizado, así como la inclusión 
Tabla 3. Medias \pm error estándar de las variables de la prueba de comportamiento y sus efectos en los marcadores para cada raza.

\begin{tabular}{|c|c|c|c|c|c|c|}
\hline Raza & Variable & Marcador & $\mathrm{p}$ & $\mathrm{n}$ & Genotipo & $\mathrm{MMC} \pm \mathrm{EE}$ \\
\hline \multirow[t]{3}{*}{ Angus } & CRA & GH-Alu & 0.032 & 29 & $\mathrm{CC}$ & $0.13 \pm 0.13^{a}$ \\
\hline & & & & 27 & $\mathrm{GC}$ & $-0.26 \pm 0.14^{b}$ \\
\hline & & & & 7 & GG & $0.48 \pm 0.28^{a}$ \\
\hline \multirow{3}{*}{ Charolais } & PFAj & & 0.041 & 18 & $\mathrm{CC}$ & $414.22 \pm 11.63^{a}$ \\
\hline & & & & 9 & GC & $362.00 \pm 16.44^{b}$ \\
\hline & & & & 5 & GG & $382.00 \pm 22.06^{b}$ \\
\hline
\end{tabular}

de nuevos indicadores productivos en las pruebas de comportamiento, son la base para que en el futuro se cuente con información fenotípica que permita la inclusión de la información genómica en los criterios de selección y mejoramiento genético.

Es importante señalar que los animales evaluados representan el primer esfuerzo en la evaluación de CRA para ganado de carne del norte de México. La principal ventaja de la integración de estas tecnologías moleculares es que una vez validadas, se espera que disminuyan los costos asociados a las evaluaciones de rasgos económicamente relevantes como el CRA. Los marcadores de eficiencia evaluados en las razas cárnicas confir- man que las frecuencias alélicas son mayores para el alelo considerado en la literatura como favorable. EI marcador $\mathrm{GH}-$ Alu presentó un efecto significativo en el CRA en la raza Angus, así como una asociación significativa con PFAj en la raza Charolais.

\section{AGRADECIMIENTOS}

Al CONACYT (294826), Instituto Politécnico Nacional (proyecto SIP-IPN No. 20151289) y productores ganaderos por su participación y consentimiento para la toma de muestras y uso de la información.

\section{LITERATURA CITADA}

Alende M (2017) Residual feed intake in cattle: Physiological basis. A review. Revista Argentina de Producción Animal 36: 49-56.

Almeida SEM, Santos LBS, Passos DT, Corbellini AO, Lopes BMT, Kirst C, et al. (2008) Genetic polymorphsims at the leptin receptor gene in three beef cattle breeds. Genetics and Molecular Biology 3: 680-685.

Banos G, Woolliams JA, Woodward BW, Forbes AB, Coffey MP (2008) Impact of single nucleotide polymorphisms in leptin, leptin receptor, growth hormone receptor, and diacylglycerol acyltransferase (DGAT1) gene loci on milk production, feed and body energy traits of UK dairy cows. Journal of Dairy Science 91: 3190-3200.

Bishop, MD,Simmen, Simmen FA, Davis ME (1989) The relationship of insulin-like growth factor-I with postweaning performance in Angus beef cattle. Journal of Animal Science 67: 2872-2880.

Buchanan FC, Fitzsimmons CJ, Van Kessel AG, Thue TD, Winkelman SDC (2002) Association of a missense mutation in the bovine leptin gene with carcass fat content and leptin mRNA levels. Genetics Selection Evolution 34: 105-116.

CONARGEN (2017) Charolais. México http://conargen.com/category/socios/bov-carne/. Fecha de consulta: 16 de abril del 2019.

Corva PM, Fernandez MGV, Soria LA, Papaleo MJ, Motter M, Villarreal EL (2009) Effect of leptin gene polymorphisms on growth, slaughter and meat quality traits of grazing Brangus steers. Genetics Molecular Research 8: $105-116$. 
Crews DH Jr (2005) Genetics of efficient feed utilization and national cattle evaluation: a review. Genetics and Molecular Research 4: 152-165.

De la Rosa, XF, Montoya, HM, Castrellon, AMS, Bracamonte, MP, Vera, WA (2010) Polymorphisms in the IGF1 gene and their effect on growth traits in Mexican Beef Cattle. Genetic and Molecular Research 9: 875-883.

Ge W, Davis ME, Hines HC, Irvin KM, Simmen RC (2001) Association of a genetic marker with blood serum insulin-like growth factor-I concentration and growth traits in Angus cattle. Journal of Animal Science 79: 1757-1762.

Geary TW, McFadin EL, MacNeil MD, Griggs EE, Short JE, Funston RN, et al. (2003) Keisler DH. Leptin as a predictor of carcass composition in beef cattle. Journal of Animal Science 81: 1-8.

Koch RM, Swiger LA, Chambers D, Gregory KE (1963) Efficiency of feed use in beef cattle. Journal of Animal Science 22: 486-494.

Llonch P, Somarriba M, Duthie CA, Haskell MJ, Rooke JA, Troy S, et al. (2016) Association of temperament and acute stress responsiveness with productivity, feed eficiency, and methane emissions in beef cattle: An observational study. Frontiers in Veterinary Science 3: 43 Doi: 10.3389/fvets.2016.00043.

Nkrumah JD, Li C, Yu J, Hansen C, Keisler DH (2005) Polymorphisms in the bovine leptin promoter associated with serum leptin concentration, growth, feed intake, feeding behavior, and measures of carcass merit. Journal of Animal Science 83: 20-28.

Pereira AP, De Alencar MM, De Oliveira HN, Almeida DR (2005) Association of GH and IG-1 polymorphisms with growth traits in a synthetic beef cattle breed. Genetics and Molecular Biology 28: 230-236.

Rousset F (2008) Genepop'007: A complete reimplementation of the Genepop software for Windows and Linux. Molecular Ecology Resources 8: 103-106.

Sherman EL, Nkrumah JD, Murdoch BM, Li C, Wang Z, Fu A, et al. (2008) Polymorphisms and haplotypes in the bovine neuropeptide $\mathrm{Y}$, growth hormone receptor, ghrelin, insulin-like growth factor 2 , and uncoupling proteins 2 and 3 genes and their associations with measures of growth, performance, feed efficiency, and carcass merit in beef cattle. Journal of Animal Science 86: 1-16.

Stick DA, Davis ME, Loerch SC, Simmen RC (1998) Relationship between blood serum insulin-like growth factor 1 concentration and postweaning feed efficiency of crossbred cattle at three levels of dietary intake. Journal of Animal Science 76: 498-505.

Yurnalis A, Putra DE (2017) Polymorphism of insulin like growth factor 1 gene (IGF1/TasI, IGF1/SnaBI, IGF1/Rsal) and the association with daily gain of Pesisir cattle local breed from West Sumatera, Indonesia. Pakistan Journal of Biological Sciences 20: 210-216.

Wood BJ, Archer JA, Van der Werf JHF (2004) Response to selection in beef cattle using IGF-1 as a selection criterion for residual feed intake under different Australian breeding objectives. Livestock Production Science 91: 69-81. 